\title{
Knowledge and attitudes of the cytology test for cervical cancer screening in a rural population in Sri Lanka - a population based survey
}

\author{
A. A. H. Priyani ${ }^{1}$, P. Thuvarakan ${ }^{1}$ and U. Senarath ${ }^{2}$ \\ Department of Pathology, Faculty of Medicine, University of Colombo, Sri Lanka ${ }^{1}$ \\ Department of Community Medicine, Faculty of Medicine, University of Colombo, Sri Lanka ${ }^{2}$
}

DOI: http://doi.org/10.4038/jdp.v11i2.7713

\section{Introduction}

Despite having a national cervical cancer(CC) screening programme, CC is still the second leading cancer among Sri Lankan females. The aim of this study was to assess knowledge and attitudes of the cytology test for CC screening in a rural population.

\section{Methodology}

HETC Rural Health Project, Faculty of Medicine, Colombo used a 2-stage stratified cluster sample of 1950 households from 13 divisional secretariats in Ampara, Monaragala, Hambanthota and Kalmunai to interview married females aged 35-65 years using an interviewer administered questionnaire.

\section{Results}

Total of 1499 females were interviewed. The mean age at marriage was 22 years. There were $1053(70 \%)$ housewives and 278(18\%) were involved in agriculture, forestry or fishery industries. Of those who disclosed ethnicity $(n=1397), 1115(80 \%), 191(14 \%)$ and $89(6 \%)$ were Sinhalese, Muslims and Tamils respectively. Of those who disclosed education level ( $n=1484), 1337(90 \%)$ had not passed ordinary level. Questions related to knowledge and attitudes of the cytology test were answered by 1233 participants. The awareness of the test in the age categories $35-40$ years $(n=311)$ and 41-45years $(n=215)$ were $249(80 \%)$ and $160(74 \%)$ respectively; the awareness decreased with the advancing age. Of 1233 participants $888(72 \%)$ have not done the test as, $448(50 \%)$ thought it was unnecessary as they did not have an illness, 306(34\%) were unaware of the facility, $69(8 \%)$ were afraid of the procedure and 69(8\%) had "practical difficulties". Cultural/ religious reasons, attitudes of parents and healthcare workers were reasons in less than $2.5 \%$. These proportions remained more or less the same for all education levels, ethnic groups and occupation.

\section{Conclusion}

The majority of the participants were aware of the screening test. However, negative attitudes resulted in failure to undergo cervical cancer screening test in a significant proportion, which can be corrected by a simple intervention like distributing a leaflet. 